CARNETS OE Carnets de géographes

GÉOGRAPHES.

9 | 2016

Géographies des émotions

\title{
Les paysages urbains en mal d'émotions
}

\section{Emeline Bailly}

\section{(2) OpenEdition}

Journals

Édition électronique

URL : http://journals.openedition.org/cdg/594

DOI : $10.4000 /$ cdg. 594

ISSN : 2107-7266

Éditeur

UMR 245 - CESSMA

Référence électronique

Emeline Bailly, «Les paysages urbains en mal d'émotions », Carnets de géographes [En ligne], 9 | 2016, mis en ligne le 30 novembre 2016, consulté le 01 mai 2019. URL : http://journals.openedition.org/ cdg/594 ; DOl : 10.4000/cdg.594

\section{(ब) $\odot \Theta$}

La revue Carnets de géographes est mise à disposition selon les termes de la Licence Creative Commons Attribution - Pas d'Utilisation Commerciale - Pas de Modification 4.0 International. 


\title{
LES PAYSAGES URBAINS EN MAL D’EMOTIONS
}

EMELINE BAILLY

Docteure en urbanisme, Chercheure au Centre scientifique et technique du bâtiment (CSTB), Chercheure associée au Lab'Urba (EA 3482 - université Paris-Est)

Emeline.BAILLY@cstb.fr

\begin{abstract}
Résumé
Les professionnels de l'urbain s'intéressent de plus en plus aux dimensions idéelles et affectives qui traversent les villes. Cette recherche du sensible symbolise la nécessité d'espaces à même d'être éprouvés, contemplés, habités dans un contexte de perte de repères urbains avec la métropolisation accélérée. Assisterions-nous pour autant à un tournant de la conception urbaine ? Rien n'est moins sûr. La considération dans les projets urbains des représentations, sensations, sentiments et émotions associés aux espaces reste à la marge. Pour appréhender le ressenti des lieux en tant que connaissance spatiale, sans les simplifier ou les instrumentaliser, il importe de s'interroger sur les définitions, les méthodes de recueil des rapports émotionnels aux lieux et leurs traductions spatiales. Les résultats des recherches-action "L'Enjeu du paysage commun " et "Fabrique Active du paysage ", nous permet d'interroger les méthodes pour appréhender les ressentis, les subjectivités partagées et surtout les potentiels descripteurs de ce que nous nommons le monde urbain sensible. La connaissance du système d'affects urbains apparaît alors comme un enjeu de conception urbaine pour des lieux et paysages urbains qui ne soient plus en mal d'émotions.
\end{abstract}

Mots-clefs : paysage, ville, paysages urbains, émotions, ressenti.

\section{Abstract}

Many contemporary urban planning professionals value affects and ideal urban dimensions. The desire for a more sensitive city symbolizes the need for spaces to be lived in, tried, contemplated and proven in the context of accelerated metropolisation. Are we witnessing a turning point in urban design? Nothing could be less sure. The consideration of representations, sensation, feeling, and emotion associated with specific urban areas still remains marginal in urban projects. To understand the feeling of a place as knowledge, without oversimplification or exploitation, we need to definite the terms and consider the emotional relationships of places, and their spatial translations, without misrepresentation. The results of the research-action "L'Enjeu du paysage commun" and "Fabrique Active du paysage" question the methodology to consider feelings, personal experience and perception and the potential descriptors of the urban sensitive world. The system of the urban affects 
knowledge challenge today's urban intervention. But there is a need to reconnect urban places and landscapes with emotion and urban planning.

Keywords: landscape, city, urban landscapes, emotions, feeling. 


\section{Introduction}

La lutte contre les formes de sentiments négatifs (insécurité, stigmatisation, etc.) ou au contraire les ambitions de préservation de "l'esprit des lieux" sont des objectifs qui pointent parmi les professionnels de la ville. Ils réintroduisent les images, sensations et même ressentis des lieux comme un fait urbain qui amène à penser une ville plus sensible. Même si elle demeure timide, cette ambition appelle à considérer les dimensions idéelles, y compris émotionnelles, dans les discours des politiques publiques. Elle symbolise la nécessité d'espaces à même d'être éprouvés, contemplés, habités dans un contexte de métropolisation accélérée.

Assisterions-nous pour autant à un tournant de la conception urbaine ? Rien n'est moins sûr. Si les discours évoluent, la considération dans les projets urbains des représentations, sensations et émotions associées aux espaces reste à la marge. Il faut dire que ce sont des objets difficiles à cerner. Ils sont immatériels et leurs ressorts renvoient à la subjectivité, aux ressentis, voire aux fantasmes. Aussi, ils restent une inconnue, faute de pouvoir être appréhendés.

Notre hypothèse est que les paysages urbains sont en mal d'émotions, dans la mesure où la conception urbaine s'interroge peu sur les sentis et ressentis associés aux lieux, sur les subjectivités partagées qu'ils peuvent susciter et encore moins sur les potentiels descripteurs urbains de ce monde sensible. Les éléments sensibles sont constitutifs des lieux qu'il s'agisse des représentations sociales, culturelles, imaginaires ou des affects (qu'il $s^{\prime}$ agisse de sensations, sentiments ou émotions comme nous allons l'expliciter). Ils font signe et permettent un ancrage spatial et temporel des individus. La recherche L'Enjeu du paysage commun nous a permis de montrer comment les sensations, sentiments, émotions et les représentations et imaginaires participaient de l'appréhension des lieux et de leurs paysages. Le paysage exprime une relation distanciée à un environnement, une possibilité d'éprouver le monde et de s'y projeter (Berque, 1996; Luginbühl, 2012 ; Bailly, 2013). Autrement dit, le paysage est ici entendu comme une expérience, la possibilité de ressentir le monde, la société humaine en relation avec sa propre existence en tant qu'être. De son vécu et expérience subjective, l'individu donne sens au lieu et peut composer son propre sentiment paysage urbain. Une absence d'évocation sensible limite la possibilité d'éprouver un paysage, une ambiance, voire un espace public. De même, les affects sentis et ressentis s'inscrivent dans les espaces publics et paysages à travers une série de signes urbains euxmêmes susceptibles d'être lus. Ainsi, selon nous et à l'instar de Denis Martouzet (2014) et de Benoit Feildel (2010), les affects, et plus particulièrement les émotions, affectent l'espace.

Comment dès lors les appréhender en tant que connaissance spatiale, sans les simplifier ou les instrumentaliser ? Comment recueillir les rapports affectifs et émotionnels aux paysages et leurs traductions spatiales sans les dénaturer? Comment questionnent-ils enfin l'intervention urbaine actuelle?

Pour aborder ces questions, nous nous interrogerons d'abord sur la place de l'émotion dans l'appréhension et le façonnage des espaces à partir des résultats de la recherche L'enjeu du paysage commun et de la recherche-action qui la prolonge, FACT. Celle-ci vise à révéler les signes urbains qui favorisent les affects dans des sites aux potentiels paysagers de L'lle-SaintDenis. Elle nous permet également de mieux distinguer les affects en présence, qu'il s'agisse des sensations, sentiments ou émotions qui affectent l'espace. Nous les mettrons en perspective avec l'apport de protocoles scientifiques et démarches participatives récentes 
s'attachant aux espaces vécus et ressentis pour interroger les pistes de connaissances et démarches urbaines plus respectueuses des rapports affectifs aux lieux et leurs paysages.

\section{Une appréhension multidimensionnelle du paysage}

La recherche L'enjeu du paysage commun s'interrogeait sur l'appréhension du paysage urbain par ses usagers dans deux quartiers périphériques en mutation, Melrose dans le Bronx et L'Ile-Saint-Denis en Seine-Saint-Denis. II en ressort, à l'instar de précédentes recherches, une considération multidimensionnelle et dynamique de l'évocation paysagère par les citadins. Les "enquêtes promenades" menées à partir de parcours commentés individuels et d'une série de dispositifs visant à saisir les perceptions, ressentis et représentations (photographies, dessins, prise de sons, cartes mentales, etc.) montrent sans surprise une hétérogénéité de rapports aux lieux et à leurs paysages. Ceux-ci sont toujours basés sur une composition urbaine, sociétale et politique, mais également sentie et ressentie des lieux. Le sensible apparaît même comme une clé de composition du rapport au paysage urbain.

\section{Des sentiments de paysage composés à partir de dimensions spatiales et idéelles}

Le paysage est abordé dans les deux terrains sans distinction entre le site naturel et les aménagements urbains, l'ici et de l'ailleurs, le statique et le mouvant. Pour les personnes interrogées, le paysage urbain n'est donc pas réduit à sa dimension naturelle mais articule le site naturel (sa géomorphologie et sa végétation) et la ville aménagée. II combine des signes proches (détails marquants, espaces archétypaux, objets emblématiques) et lointains, les mouvements naturels (lumières, vents dans les arbres,...) et des flux ou les rythmes urbains (cadences des transports en commun...). Ces signes sont porteurs de sens et permettent à chacun de se situer et d'éprouver sa présence dans le lieu, voire dans le monde.

Le paysage est également appréhendé à travers les signes de ses évolutions urbaines à l'interface du passé (héritage), du présent (appropriation) et du futur (bâtiments en construction, projection). La sédimentation des traces au fil du temps, leur caractère aléatoire, l'univers de "bric et de broc ", voire imparfait qu'elle crée, est valorisé par les personnes interrogées. A l'inverse, certains projets d'urbanisme récents suscitent de l'inquiétude. Ils sont perçus par une majorité des résidents interviewés, notamment les personnes actives, comme l'expression d'une "norme " urbaine, à l'image de celle des villes centres, qui « lisserait » les lieux, les rendraient « froids » et difficilement appropriables. Par exemple, un quai cimenté et aménagé en bord de Seine en remplacement d'un chemin de terre bordé d'une végétation sauvage est considéré "sans âme", "aseptisé ". La perception d'un ensemble constitué d'époques différentes participe ainsi du paysage temporel, c'est-à-dire d'une possibilité d'inscription dans le devenir humain du monde.

Le paysage est valorisé par ses usagers en tant qu'il est habité comme l'avait déjà montré John Brinckerhoff Jackson (1984). Plus que ces usages, les interviewés soulignent l'importance de la visibilité des autres citadins. Le sentiment de coprésence, c'est-à-dire de perceptibilité mutuelle (Goffman, 1963), révèle l'espace urbain chargé de la vie des hommes, de leurs relations subjectives, des valeurs, expériences et imaginaires qu'ils confèrent au lieu. La perception des autres permet de se situer dans une sphère sociale, $y$ compris dans un rapport à l'altérité. II ouvre une conscience d'être un parmi les autres. La 
composition entre soi, autrui et un lieu semble représenter alors une alliance incontournable pour appréhender des paysages en milieu urbain. Le paysage habité traduit en effet un sentiment d'appartenance à l'humanité (Berque, 1994).

Les représentations sociales et culturelles des lieux (emblème, métaphore, imaginaires) et souvenirs intimes irriguent les discours des interrogés. Ceux-ci fondent un rapport idéel au paysage. Ils varient selon les référents sociaux, culturels, ou personnels, mais permettent aux interrogés d'inscrire leur appartenance à une vie intime, de voisinage, social, sociétal. Ainsi, l'individu apparaît spatial, autant que l'espace apparaît humanisé.

La transformation du paysage politique, c'est-à-dire du paysage issu des interventions de l'action publique (composition urbaine, aménagement, végétalisation, etc.) ou des initiatives locales, joue modérément dans l'appréciation du paysage même si, quand ils sont interrogés, les usagers préconisent une série d'améliorations urbaines. Par contre, les personnes interrogées insistent sur leurs désirs d'implication dans l'évolution du territoire et de ses paysages et valorisent un paysage vernaculaire de voisinage, fruit des microtransformations spatiales de chacun. Elles se sentent souvent peu entendues, pour ne pas dire dessaisies de ce qui constitue leur lieu de vie, c'est-à-dire de l'espace vécu, expérimenté, représenté. C'est une véritable question posée aux politiques publiques.

Au-delà du paysage urbain, temporel, politique et habité, sensations, sentiments et émotions semblent constituer un des principaux leviers du sentiment paysager. Ce dernier apparaît plus diversifié à L'lle-Saint-Denis, n'étant pas dominé par des sentiments d'inquiétude face à l'insécurité comme à Melrose dans le Bronx (Bailly et al., 2014).

Dans les deux sites, les sensations s'expriment à travers les cinq sens mais aussi le mouvement qui, pour Alain Berthoz (1997), constitue un sixième sens. Si la vue prime, les sons, l'odorat sont appréciés, notamment en lien avec la nature (oiseaux, bruissement des arbres, odeur de la terre, etc.) ou des imaginaires (par exemple, le voyage des avions). Le toucher est évoqué par détour pour exprimer une sensation plaisante (fraîcheur, brise, courant d'air, etc.), mais aussi pour exprimer la sensation de son corps (marche, poids du corps sur un sol meuble). Plus largement, c'est le sentiment de se sentir vivant en lien avec le milieu ambiant qui est mis en avant. II favorise non seulement des sentiments de paysages mais aussi d'apprécier son ambiance (Augoyard, 2011 ; Bailly et al., 2014).

Les sentiments, bien que subjectifs et variables, sont omniprésents. Ils autant positifs que négatifs. Ils prennent d'abord la forme de sentiments généraux (attachement, appartenance, ancrage, satisfaction) ou esthétiques (beau, raffiné, etc.). Ils sont également inspirés par l'environnement, que ce soit la nature (liberté, évasion, lien à la terre, refuge), l'eau (quiétude, calme, apaisement, tranquillité) ou encore la ville lointaine (plaisir, admiration, etc.). En revanche, peu de sentiments apparaissent associés à l'espace urbain proche, si ce n'est négatif (colère face à l'isolement d'un ensemble d'habitat, à l'abandon public de « cités sans vie ", "isolées", aux délaissés urbains qui créent des "déserts "). Sensations et sentiments suscitent enfin des émotions, ce qui traduit un état éprouvé à la fois par le corps et l'esprit comme par exemple la joie.

Ces affects (qui se déclinent sous forme de sensations, sentiments et émotions) apparaissent essentiels au senti et ressenti paysager, et ce en lien avec des configurations d'espaces, à savoir l'offre de points de vue distanciés, d'un horizon, d'un champ de vue élargi. Ils créent ce que nous nommons le paysage ressenti. 
Le paysage exprime ainsi un ensemble de relations des hommes aux lieux selon plusieurs dimensions (urbain, habité, temporel, politique) tel que l'avait déjà établi John Brinckerhoff (1984) ou Yves Luginbühl (2012). Il est aussi lié aux affects éprouvés, autrement dit ressentis. Les interdépendances entre expérience, représentation, perception et ressenti permettent un ancrage territorial aussi bien distancié qu'immersif, personnel que collectif, affectif qu'objectif. Globalement, le paysage apparaît appréhendé, hiérarchisé et interprété par chacun différemment. II engage notre subjectivité, nos représentations sociales et culturelles, mais aussi nos sens et affects. II transpose ainsi une appréhension subjective en une expérience collective, ce que nous nommons le paysage commun, c'est-à-dire apprécié et ressenti par tout un chacun en parallèle dans une dynamique d'intersubjectivité.

\section{Ressentir le monde, clef de composition du paysage urbain et du rapport aux lieux}

Le ressenti apparaît comme une clé de composition du paysage urbain perçu. II permet d'éprouver les lieux mais aussi de modérer ou nier ce qui est moins signifiant voire apprécié négativement. Il permet une personnalisation de la relation aux lieux et une expérience partagée du sentiment de paysage. II permet d'expérimenter la relation permanente entre les lieux et les humains, l'esprit des lieux, la symbolique qu'ils portent en eux (Foucault, 1967). Il apparaît empreint de cosmologie (en tant que rapport à la terre, à la nature, au ciel, aux hommes) et de poétique (rêver sa présence sur terre, sa vie collective pour mieux se projeter vers la mort). Augustin Berque, dans son ouvrage Etre humain sur terre (1996), pense d'ailleurs que l'homme n'a jamais cessé de penser cosmologiquement le monde et d'en symboliser le sens. La ville, en tant que milieu, est l'empreinte de l'action des hommes, de leur rêverie, mais aussi la matrice de leur subjectivité. Pour Augustin Berque, chaque lieu engage l'existence des humains et nier les lieux, c'est nier l'existence humaine dans son lien à la terre, "porter atteinte à la ville, c'est porter atteinte à des êtres humains " ( $p 156)$. Ainsi, cette matrice de subjectivité apparaît au cœur de la relation entre les hommes et leurs environnements, y compris émotionnellement.

Le ressenti paysager exprime ainsi une relation sensible et distanciée à un environnement. II offre une possibilité d'éprouver le monde et de s'y projeter (Berque, 1996; Luginbühl, 2012 ; Bailly, 2015). Le sentiment de la coprésence des hommes attaché à l'appréhension de l'espace public traduit le territoire de l'activité et de la rencontre des hommes, de la représentation et de la projection sociétale et culturelle. La sensation est enfin au cœur de la perception des ambiances (Bailly et al., 2014) qui traduit une expérience sensorielle, l'immersion dans un lieu (Augoyard, 2011). Dès lors, la relation aux espaces urbains apparaît liée aux affects. Ceux-ci permettent d'éprouver les paysages, de vivre les espaces publics et de sentir les ambiances. En retour, ils affectent l'espace. Ils peuvent être traduit en mots, en images, impressions mais aussi spatialement. Ils s'ancrent à travers un ensemble de traces, signes, imaginaires, symboliques. Ils créent selon nous un langage à même de susciter une lecture sensible des lieux et paysages.

\section{Les émotions affectent l'espace}

Si le ressenti façonne l'espace et ses paysages, reste à savoir comment le considérer dans la pensée urbaine. En effet, l'appréhension du monde sensible qui nous environne a été délaissée au profit des approches rationnelles. Or, ce monde sensible nous envahit. Nous le 
sentons, le ressentons à défaut de l'objectiver.

\section{Les émotions comme fait social et spatial}

Le sensible dans le champ urbain demeurait jusqu'à peu mal connu. Nous proposons de nous interroger sur les termes et méthodes déployés dans des recherches récentes pour tenter de le cerner.

Il importe d'abord de distinguer le sentir du ressentir. Le sentir renvoie aux sensations éprouvées par les cinq sens. Le ressenti est lié aux sentiments, à leurs interprétations par l'intellect ou l'inconscient et ce, en fonction de ses références personnelles, sociales, culturelles et intellectuelles (Martouzet, 2013), mais aussi aux émotions que ces sentiments et sensations procurent corporellement et mentalement. Sentir et ressentir (lié à l'éprouver) créent ce que nous qualifions le monde sensible. Les sensations, sentiments et émotions en composent le système d'affects (Figure 1 ) qui sont éprouvés et traduits dans les lieux et paysages sous la forme d'un langage sensible.

Figure 1. Le système d'affects à l'origine du monde sensible

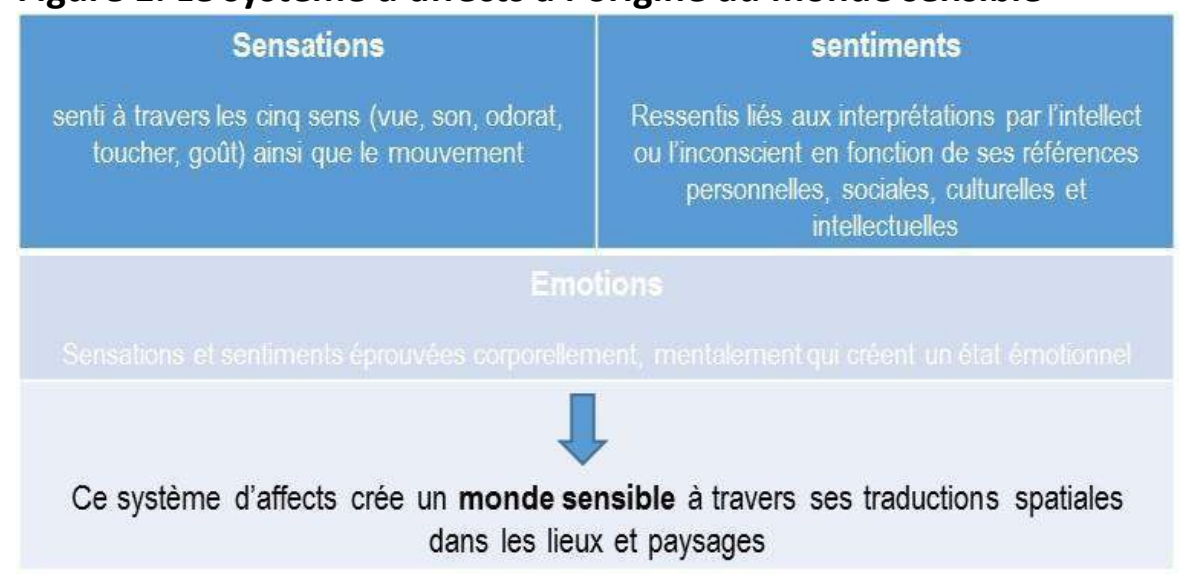

Source : 2016, E. Bailly, Cstb

Les émotions en constituent l'acte le plus visible dans la mesure où elle crée une manifestation physique à un moment donné, dans un mouvement et un lieu. Elle crée un état émotionnel chez l'individu. Au-delà du débat entre naturalistes (pour lesquels l'émotion est innée et universelle) et béhavioristes (qui y voient une réaction corporelle à des stimuli extérieurs), l'émotion résulte, selon nous, de l'interface entre des perceptions, des sensations physiques et des évaluations socio-cognitives. Cette dimension cognitive introduite par la psychologie dans les années soixante a permis son analyse dans les sciences humaines (Zirotti, 2010 ; Bernard, 2015). Les émotions sont alors étudiées en tant que convention culturelle, phénomène de socialisation qui participe des faits sociaux. Par exemple, lors d'un enterrement, une pression morale s'exerce pour manifester de la tristesse comme signe d'appartenance à la cérémonie collective. Les sociologues s'attachent à son rôle dans la communalisation, c'est-à-dire l'appartenance à une communauté (Weber, 1922), dans l'incorporation du social par des habitus émotionnels reproduisant des schémas émotionnels sous forme d'affect (Bourdieu, 1987).

En analogie avec le fait social total, Marcel Mauss (1934) introduit la notion de l'homme total (biologique, psychologique et sociologique). L'émotion renvoie au sujet socialisé 
affectivement et socialement influencé. Ainsi, deux approches des émotions coexistent, l'une où le groupe social prime, l'autre où c'est l'individu.

Les anthropologues et sociologues (Dewey, 1929 ; Boltanski, 1993 ; Livet et Thévenot, 1993) proposent un cadre théorique plus général de l'homme, liant la sensation et la cognition, le sentiment et la raison, le perceptif et l'interprétation. Surtout, ils considèrent que les affects imprègnent l'homme en société et ne peuvent se réduire à une fonction sociale. Selon nous, l'émotion apparaît en effet irriguée autant par l'intellect, l'inconscient, les sentis et ressentis. Elle anime tout un chacun individuellement et collectivement, notamment dans son rapport à l'espace. Plus encore, dans notre conception, elle affecte l'espace.

La considération du sensible dans le champ urbain reste pour autant secondaire, les affects restant associés aux individus et non aux lieux. Pourtant, plusieurs disciplines montrent l'existence d'un espace affectif et peuvent constituer un premier socle conceptuel pour aborder le monde sensible qui anime les territoires urbains. Les historiens rapprochent l'esthétique avec des styles émotionnels variant selon les époques (Reddy, 2001). L'anthropologie et la sociologie urbaines s'intéressent à la vie nerveuse des villes liées aux impressions en présence (Simmel, 2013), aux émotions suscitées par les formes urbaines (Ledrut, 1984) et aux images et imaginaires urbains qu'elles génèrent. Les psychologues de l'environnement, tels Yi-Fu Tuan (1977) ou Jean-Paul Thibault (2015), insistent sur l'importance de l'inscription spatiale et des formes d'attachement (configuration spatiale et lien social) et les savoir-faire individuels développés pour éprouver (ralentir, s'arrêter, regarder, etc.).

La recherche L'enjeu du paysage commun poursuit cette nécessité de comprendre comment le ressenti affecte l'espace et la qualité des lieux elle-même. Articuler sciences sociales et spatiales, ressentis individuel ou collectif et charges sensibles des lieux nous apparaît dès lors comme un enjeu pour la recherche urbaine.

\section{Articuler monde sensible et espace}

Des recherches récentes ont d'ores et déjà amorcé l'articulation entre les territoires et le sensible. Ainsi, un ensemble de descripteurs (Figure 2) peuvent permettre d'approcher ce que nous nommons la charge sensible des lieux. James J. Gibson (1979) s'intéresse aux possibilités et opportunités qu'offrent les lieux, ce qu'il nomme l'affordance. Raymond Ledrut (1984) estime que la forme outre sa matérialité (matière, consistance) n'existe que parce qu'elle a du sens qui se sent, et en conséquence appartient autant au monde sensible que physique. Denis Martouzet et Benoit Feildel (2013) s'intéressent aux ressentis individuels et aux prises matérielles qui les suscitent. Ils parlent de prise affective à même de favoriser un espace urbain affectif. William Reddy (2001) considère les signes que constituent les émotions elles-mêmes, qu'il nomme les émotives. Celles-ci décrivent l'espace affectif vécu. Notre recherche souligne l'importance des marques sensibles liées aux sédimentations d'expériences subjectives et aux traces laissées dans les lieux.

Parallèlement, des enquêtes explorent le rapport affectif aux lieux (Feildel, 2013 ; Martouzet, 2014 ; Thibault, 2015). Julien Bernard (2015) identifie des marqueurs émotionnels visibles dans les mots et les attitudes des individus. II peut alors devenir possible d'envisager identifier les subjectivités partagées, C'est-à-dire une émotion partagée face à lieu, un paysage et le passage d'un ressenti individuel à une reconnaissance collective, 
(Aubry, 2006), cette capacité commune à ressentir un lieu simultanément.

De même, la représentation sensible des lieux est explorée. Henry Pierre Jeudy (2003) s'intéresse au récit fictionnel du promeneur issu de son expérience intellectuelle et sensible tandis que Pierre Sansot (2004) insiste sur la puissance de la relation affective et imaginaire des hommes par rapport à une ville. Gilbert Durant (1996) parle de "zones de haute pression imaginaire " (p. 17) susceptibles d'être identifiées par une mytho-analyse fondée sur une approche historique et une géographie des mythes.

\section{Figure 2 : Exploration des descripteurs possibles du monde sensible}

\begin{tabular}{|c|c|}
\hline \multicolumn{2}{|c|}{ Rapports affectifs aux espaces } \\
\hline $\begin{array}{l}\text { Senti etressenti } \\
\text { (Bailly 2015) }\end{array}$ & $\begin{array}{l}\text { Système d'affects (sensations, sentiments, } \\
\text { èmotions) exprimés }\end{array}$ \\
\hline $\begin{array}{l}\text { Marqueurs emotionnels } \\
\text { (Bernard, 2015) }\end{array}$ & Visibles dans les mots, attitudes des individus \\
\hline $\begin{array}{l}\text { Subjectivites partagées } \\
\text { (Aubry.2006) }\end{array}$ & $\begin{array}{l}\text { Capacité commune à ressentir un feu ou } \\
\text { expérience intersubjective } \\
\text { partage du sensible (Rancière, } 2000 \text { ) }\end{array}$ \\
\hline \multicolumn{2}{|c|}{ Representations sensibles: } \\
\hline $\begin{array}{l}\text { Puissance de representation } \\
\text { imaginaire dune ville } \\
\text { (Sansot, 2004) }\end{array}$ & $\begin{array}{l}\text { Reprèsentations affectives et imaginaires dune } \\
\text { vile }\end{array}$ \\
\hline $\begin{array}{l}\text { Recit fictionnel } \\
\text { (Jeudy, 2003\} }\end{array}$ & $\begin{array}{l}\text { Réci fictionnel du promeneur issu de son } \\
\text { experience intellectualle et sensible }\end{array}$ \\
\hline $\begin{array}{l}\text { Zone de haute pression } \\
\text { imsginsire } \\
\text { (Durant,1986) }\end{array}$ & $\begin{array}{l}\text { Géolocalisation des lieux évocateurs de mythes et } \\
\text { imaginaires }\end{array}$ \\
\hline Paysage ressenti (Baily, 2014) & $\begin{array}{l}\text { Il peut être éprouvé si existe une composition de } \\
\text { descripteurs de la charge sensible des lieux, de }\end{array}$ \\
\hline \multicolumn{2}{|c|}{ Charge sensible des libux } \\
\hline $\begin{array}{l}\text { Affordance } \\
\text { (Gloson, } 4979)\end{array}$ & Possibilités et opportunités qui offre les feux. \\
\hline $\begin{array}{l}\text { Forme de respace } \\
\text { (Ledrut, 1084) }\end{array}$ & $\begin{array}{l}\text { Forme dans sa matérialité (matière, consistance) } \\
\text { et dans son sens (signification, sensation) }\end{array}$ \\
\hline $\begin{array}{l}\text { Marques sensibles. } \\
\text { (Bailly et AL, 2015) }\end{array}$ & $\begin{array}{l}\text { Traces liées aux sédimentations d'expériences } \\
\text { sensibles dans les lieux }\end{array}$ \\
\hline $\begin{array}{l}\text { Prises affectives } \\
\text { (Whartouzet et Feildel (2013) }\end{array}$ & $\begin{array}{l}\text { Prises matérielles sourcas d'affects dans les } \\
\text { territcires: }\end{array}$ \\
\hline $\begin{array}{l}\text { Emotives } \\
\text { (Whiliam Reddy, 2009) }\end{array}$ & Signes que constbuent les émotions eles-mâmes \\
\hline
\end{tabular}

Source : 2016, E. Bailly, Cstb 
Le monde sensible pourrait dès lors s'appréhender par l'étude des affordances spatiales, du sens des formes, des prises affectives, des ressentis exprimés et des attitudes pouvant les traduire (marqueurs), des émotives ainsi que des fictions et imaginaires urbains associés. Ces descripteurs permettraient ainsi de traduire une partie de la réalité du monde sensible, tant spatial qu'idéel, et d'établir cette relation complexe entre le visible et l'invisible. Ils pourraient permettre de comprendre et dessiner la ville éprouvée et non seulement celle rationnelle. Cette ville éprouvée serait alors liée à l'expérience émotionnelle des lieux qui favoriserait sa constatation, sa manifestation et son interprétation dans un partage du sensible (Rancière, 2000). Elle pourrait révéler la réalité du monde tel que nous le vivons, l'expérimentons concrètement, en tant qu'expérience du monde et non telle que la figure la rationalité scientifique (Berque, 1996).

\section{La recherche urbaine à l'épreuve du sensible}

La connaissance du monde sensible urbain s'est esquissée à travers la critique de l'objectivisme. De Bergson à l'école de Chicago, de Merleau Ponty à Maldiney, de Pérec à Sansot, la question du sensible s'est perpétuée dans la pensée urbaine sans pour autant influer sur l'intervention urbaine.

\section{Résistances à l'approche du sensible}

La considération du monde sensible urbain reste globalement peu traduite méthodologiquement et opérationnellement. Elle pose en effet des difficultés théoriques, méthodologiques et même éthiques. $N^{\prime} y$-a-t-il en effet pas un risque à instrumentaliser le sensible en créant des "experts du sensible ", qu'ils soient artistes ou professionnels de l'urbain ? Ne faut-il pas au contraire laisser ce monde sensible dans une zone d'ombre ? La question reste ouverte même si des recherches récentes tentent de l'explorer.

De fait, de nombreux auteurs se sont érigés contre le risque d'instrumentalisation du sensible. Pierre Sansot (2004) estime que les affects échappent à toute méthodologie scientifique. Par exemple, le recours aux publicités ou le design émotionnel joue sur l'image iconique et les émotions pour susciter l'attractivité des territoires. De même, Raymond Ledrut (1984) estime que le sensible ne peut être typifié, le sensible étant "étranger à tous classements, il est de l'ordre de la qualité pure " (p. 11). Pour autant, il propose de lier la morphologie urbaine à la sémiologie pour créer un langage du sens formel. Or, le sens est, selon nous, pénétré du sensible.

Dès lors, relier l'intelligible et le sensible ne peut-il s'envisager à partir d'un appareil conceptuel transversal ? Ce sont les mises en relation des dimensions physiques et idéelles qui semblent permettre de concilier appréhension subjective et objective. Ce sont les interfaces elles-mêmes qui semblent à même de créer des ressentis spatiaux, leurs interprétations intellectuelle, émotionnelle et imaginaire mais aussi la charge sensible des lieux. Ainsi, il s'agit d'établir une pensée relationnelle indépendamment d'un quelconque "fond » fixe et immuable (l'espace ou l'individu, l'objectif ou le subjectif) - et dynamique tenant compte de la recomposition en continu du monde sensible en reconstruction/déconstruction permanente. 


\section{La recherche peut-elle analyser le monde sensible?}

Des recherches récentes explorent certaines dimensions du monde sensible et des méthodologies pour les appréhender (Blanc, 2012; Martouzet, 2013 ; Manola, 2013 ; Feildel, 2010 et 2013). Elles permettent progressivement le déploiement d'un corpus scientifique sur la connaissance du monde sensible urbain. Par exemple, Nathalie Blanc et Jacques Lolive (2013) préconisent de donner un statut au paysage ordinaire en tant que vecteur d'esthétique environnementale tant visuel qu'immersif. Ils considèrent en effet, à la suite d'Arnold Berleant (1990), que ce qui caractérise l'esthétique environnementale actuelle " $n$ 'est pas la contemplation désintéressée, mais l'engagement total, une immersion sensorielle dans le monde naturel [...] une expérience de l'unité exceptionnelle " (p. 235). Cela les conduit à suggérer des démarches participatives plus sensorielles, croisant approches artistiques et scientifiques en vue d'articuler l'écologie et l'esthétique, de renouveler les représentations de la nature et d'enrichir l'espace public et politique. II s'ensuit des expériences de création croisant art et environnement, telle que celle proposée par l'association COAL (Coalition pour l'art et le développement durable).

\section{FACT : Une recherche-action qui interroge le monde sensible urbain}

Dans ce contexte de questionnements méthodologiques, et fort des résultats sur l'importance du sensible dans l'appréhension du paysage urbain, nous avons initié une nouvelle étape de recherche: Fabrique ACTive du paysage (FACT). L'enjeu était d'approfondir nos premières hypothèses de recherche sur les subjectivités partagées et les descripteurs du monde sensible, mais aussi de développer des protocoles de recueil du senti et ressenti urbains moins susceptibles d'être déployés dans des projets urbains. La méthode très qualitative mobilisée pour la première étape de recherche L'enjeu paysage commun s'appuyait sur des enquêtes promenades en face à face. Elle a permis de recueillir l'hétérogénéité d'appréhensions des lieux, y compris ressenties, et l'attention aux marques et signes sensibles des lieux. Des ressentis individuels ont pu être mis en perspective les uns avec les autres et des subjectivités partagées identifiées pour plusieurs lieux. Ces méthodes étaient riches d'enseignements qualitatifs, mais nécessitent un investissement dans le temps qui n'apparaît pas compatible avec les exigences urbaines opérationnelles.

La mise à l'épreuve de ces résultats dans le cadre de la recherche-action $\mathrm{FACT}$, vise à révéler huit sites repérés comme stratégiques. Il s'agit de recueillir ce qui fait sens, sensations, sentiments et émotions et de mettre en exergue des descripteurs spatiaux ou sensibles contribuant au ressenti paysager. Un nouveau protocole d'enquête, dit d'atelier promenade, a été élaboré en ce sens. II vise à recueillir, dans un temps limité (environ trois heures), le rapport aux lieux et à leurs paysages par une douzaine d'usagers. Chaque atelier promenade (Figure 3) articule temps individuel et collectif. Le temps individuel étudie les ressentis et appréhensions du paysage à travers une succession de mises en situation et de questions dont les réponses sont écrites dans un carnet. Chacun peut ainsi explorer, in situ et en solitaire, ses perceptions (description du lieu et des éléments marquants), ses sensations (prises de photographies, captations de son, dessins, textes), ses sentiments (positionnement dans l'espace en fonction de ses affects), ses émotions (état ressenti), expériences et représentations sociales ou imaginaires, et les synthétiser par écrit et le dessin d'une carte mentale. 
Figure 3 : Atelier promenade à L’Ile-Saint-Denis, 2014


Source : 2014, Cstb

La confrontation de différents matériaux permet d'identifier ressentis et descripteurs urbains du sensibles (signes, symboliques, etc.). La deuxième séquence, collective, vise à transcrire et confronter l'ensemble des ressentis et représentations sensibles recueillis et leurs marqueurs spatiaux sur une carte commune du site. Elle explore également au moyen d'une carte mentale collective les valorisations possibles du site et de ses paysages sensibles.

Ce protocole d'atelier promenade confirme les compositions paysagères individuelles et l'importance du ressenti dans l'appréhension des lieux. Il permet, via un dispositif plus léger que l'enquête promenade, de traduire des affects urbains et leurs traductions spatiales. Plus le sens de l'espace est perçu et ressenti, plus il crée de subjectivité partagée, plus un sens commun s'établit. Pour autant, en raison du petit nombre de personnes mobilisées, l'identification des ressentis reste partielle de même que les descripteurs de la charge sensible des lieux. Ce principe d'atelier promenade apparaît prometteur mais demanderait dès lors à être testé sur des échantillons plus importants. Cette expérience apparaît intéressante pour saisir l'ancrage affectif dans un lieu et son paysage, mais elle demanderait à être mise en perspective avec d'autres protocoles sur le rapport affectif et imaginaire de l'environnement urbain élargi.

A l'instar de l'appréhension de la ville aimable par une diversité de dispositifs d'enquête (Martouzet, 2014), une mixité de protocoles semble nécessaire pour saisir ce monde sensible.

\section{La recherche peut-elle favoriser des projets urbains plus respectueux du monde sensible?}

La réception par les professionnels de l'aménagement de ces méthodes de recueil du sensible reste à construire. Ceux-ci valorisent les postures d'expert, au service d'une d'objectivation et d'une maîtrise rationnelles du réel. Ils masquent les choix urbains motivés par leurs propres projections imaginaires ou émotionnelles (Bailly, 2009). Ils tendent ainsi à déréaliser la relation des hommes aux lieux.

Des enjeux urbains récents favorisent les oxymores de ville attractive ou aimable, tels la ville créative, érigée en modèle par Richard Florida (2005). Mais c'est avant tout pour favoriser l'attractivité des métropoles pour les classes créatives, cette population d'actifs diplômés et flexibles susceptibles de contribuer au développement de l'image territoriale et de 
l'économie locale. Cette ville créative instrumentalise le sensible pour des enjeux de marketing sans rapport avec ce que pourrait constituer la charge sensible et ressentie d'une ville éprouvée.

\section{FACT: Une recherche-action qui questionne la traduction spatiale de la connaissance sensible des espaces urbains et de leurs paysages}

Pour mieux comprendre cette difficulté de prise en compte du sensible par les professionnels de l'aménagement, nous avons voulu tenter une expérimentation, dans le cadre de la recherche action FACT. En effet, une mise en situation a été initiée en partenariat avec les collectivités concernées, un collectif d'architectes (Bellastock) et la D.R.A.C. Elle s'appuie sur des interventions urbaines et artistiques pour chaque site. Elle vise à accentuer ce qui fait signe et révéler la charge sensible des lieux et paysages. L'appel à candidature des concepteurs met en perspective les résultats de l'enquête promenade, les ateliers promenades comme fondement de l'intervention. Ceux-ci ont été sélectionnés notamment sur leur capacité à faire résonner le sensible des lieux, à mobiliser les usagers et à proposer des interventions douces ménageant les potentialités spatiales. Notre hypothèse était d'explorer, avec ses concepteurs, un langage urbain à partir des descripteurs du monde sensible et d'en favoriser leurs lisibilités afin de multiplier les possibilités de composer un rapport à l'espace propre à chacun et, par extension, d'éprouver les lieux en commun. Après le partage de la connaissance des signes et prises affectives identifiés, des concepteurs et artistes ont ainsi été invités à les révéler et à les mettre en exergue par une approche plastique, spatiale et idéelle. Ils étaient incités à souligner en douceur, c'est-à-dire de manière discrète, des détails, points de perspective, éléments symboliques, etc. d'un site à partir d'une connaissance des descripteurs sensibles ou au contraire de favoriser l'arrêt (Figure 4) et la contemplation (Figure 5) dans un lieu évocateur de paysage. 
Figure 4 : Renaturation et banc d'observation

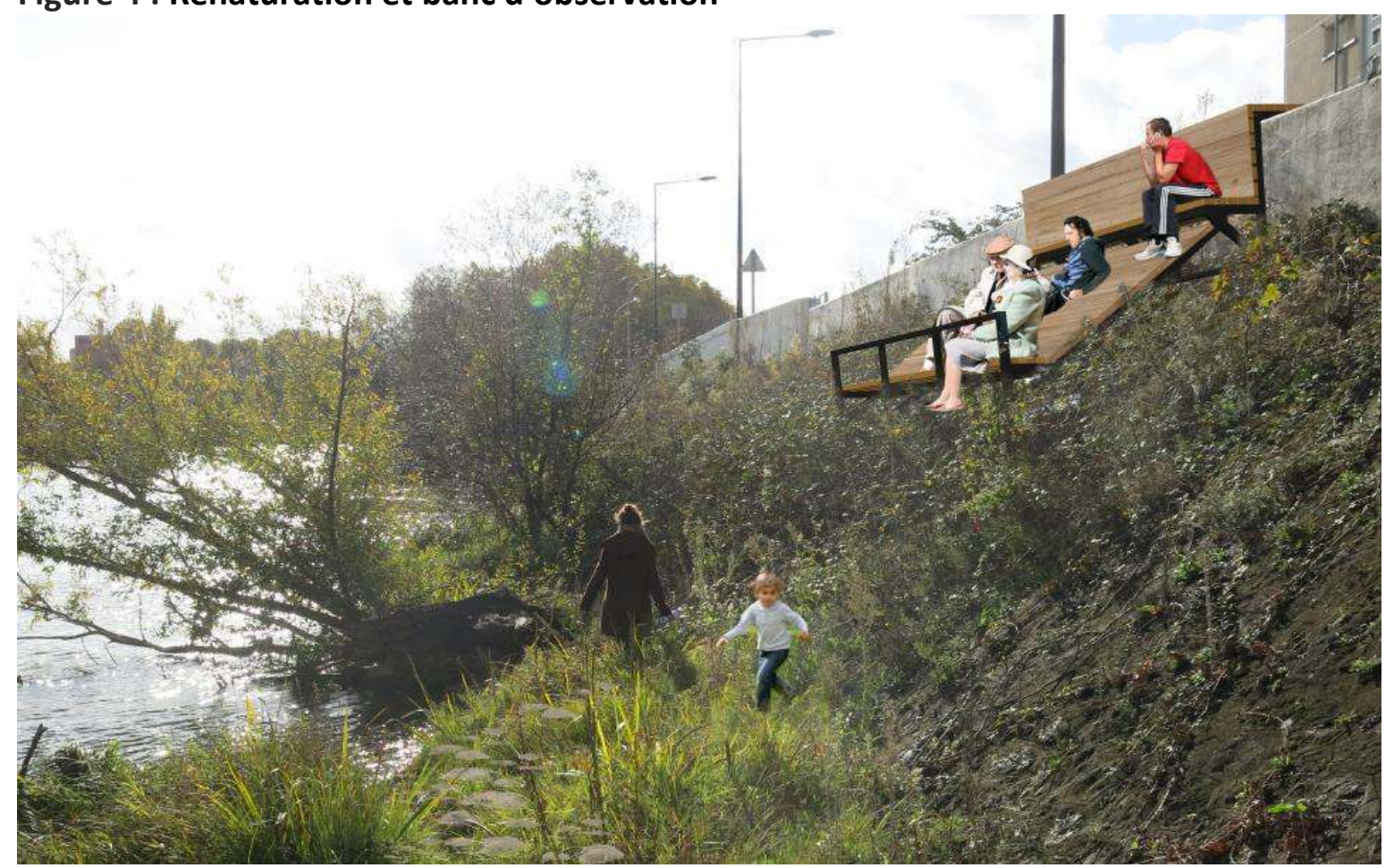

Source : 2015, Bellastock - Cstb.

\section{Figure 5 : Contemplation sur l'eau}



Source : 2015, Fabrique de l'est.

La mobilisation artistique pour participer à cette initiative a été importante. Si les projets 
retenus permettent de révéler des lieux, d'en favoriser l'expérience et la sensation par les sens, ils ne développent qu'à la marge l'idée d'un langage sensible du paysage à partir des descripteurs sensibles identifiés. Les concepteurs et partenaires ont rapidement opéré une réduction du matériau des enquêtes promenades. Les artistes des quatre premiers sites investis tendent à revisiter les lieux et paysages à travers leur propre sensation ou projection spatiale, et non celles des usagers, pour concevoir leur intervention. Les professionnels et élus ne cessent, quant à eux, de revenir sur les questions de réglementation, de gestion et de sécurisation des projets. Le plus souvent, l'enjeu d'explorer la perspective d'une conception urbaine plus sensible est délaissé au profit d'une centration sur l'action (transformation spatiale, modalités de mises en œuvre, autorisation, etc.).

Cette difficulté peut s'expliquer par les problèmes de communication entre chercheurs et praticiens, mais aussi en raison de présupposés et pratiques professionnelles qui, lors du passage à l'acte, freinent les changements de postures. Ainsi, notre observation participante active il nous semblait, en effet, qu'une implication dans l'intervention nous permettrait de comprendre de l'intérieur les blocages et leviers de changement témoigne du difficile passage de résultats de recherche vers l'action.

Pour la deuxième série de sites à investir à L'lle-Saint-Denis, ne faudrait-il pas, dès lors, impliquer les concepteurs artistes dans la phase d'atelier promenade afin d'éviter des interventions urbaines qui légitiment le discours d'un artiste ? Ils seraient ainsi, eux aussi, invités à partager in situ ce monde sensible avec les citadins, afin de dévoiler ces multiples signes et les mettre en langage. Ne faudrait-il pas encourager la phase esquisse comme une phase de recherche d'un langage commun avant d'entrer dans la formation d'un projet d'intervention qui conditionne ensuite une série de questions de faisabilité ? Dans tous les cas, cette difficulté pose plus particulièrement la question de la traduction opérationnelle de cette connaissance du monde sensible.

Cette recherche-action interroge le statut de la connaissance du monde sensible. Faut-il la réserver au champ de la recherche, pour limiter les risques d'instrumentalisation, de réduction à quelques interprétations stéréotypées ? Faut-il laisser libre l'interprétation du concepteur qui recourt à ses propres perceptions, au risque qu'elles soient complètement en opposition avec celles des résidents ? Faut-il au contraire poursuivre cette exploration du monde sensible qui affecte l'espace et qui pourrait ouvrir des perspectives de conception urbaine plus respectueuse du rapport que les hommes entretiennent avec les lieux et leurs paysages ? Selon nous, ce chemin de la recherche nécessite d'être poursuivi et, là aussi, la multiplication des expérimentations pourrait permettre, il nous semble, de dessiner un chemin vers une conception urbaine plus sensible.

\section{Pour conclure}

L'appréhension du monde sensible dans le champ urbain reste une problématique émergente. Plusieurs obstacles se combinent entre un cadre théorique en construction, des méthodologies qui restent exploratoires et peu transposables vers le monde professionnel. Une série de recherches tant théoriques, conceptuelles que méthodologiques semble encore nécessaire pour en préciser les contours et expliciter les descripteurs sensibles qui affectent l'espace et étudier la manière de les révéler ou au contraire les protéger pour éviter leur instrumentalisation. Mais, comme on a pu le voir, le retour du sensible dans la 
recherche urbaine et son exploration empirique dessine d'ores et déjà de nouvelles perspectives prometteuses.

Par ailleurs, à la marge des politiques urbaines, le mouvement dit du tactical urbanism (urbanisme tactique), selon Mike Lyndon (2010), revendique des ruses et tactiques de résistance, en référence à Michel de Certeau (1990), pour détourner les objets et les codes et se réapproprier l'espace. Les interventions des tactical urbanists cherchent à activer de nouvelles manières d'être en ville respectueuses des désirs des citadins. Elles valorisent l'imaginaire et l'inventivité habitantes plutôt que la rationalité exogène. Elles disent hybrider interventions architecturales artistiques et culturelles pour créer de nouveaux espaces collectifs plus vivants, plus expérientiels. Elles explorent ainsi de nouvelles pratiques urbaines plus qualitatives même si elles ne réussissent pas à s'inscrire dans des démarches pérennes et globales. Centrées sur des échelles de proximité, voire des microsites, elles demeurent le plus souvent ponctuelles et ne réussissent pas à infuser les échelles urbaines.

Ainsi, la question du sensible réémerge tant du côté de la recherche que des professionnels de l'urbain. Elle traduit, il nous semble, le besoin d'une ville plus respectueuse des êtres dans leur rapport émotionnel, sensoriel et sentimental aux lieux et paysages urbains. Elle traduit le besoin d'une ville susceptible d'être rêvée, ressenties et éprouvée, de liens poétiques des citadins à leurs territoires. Elle peut nourrir la perspective d'une conception urbaine sensible que l'urbanisme rationnel semble avoir délaissé.

\section{Bibliographie}

Aubry P. (2006), "Subjectivité partagée ", in Berque Augustin (dir.), Mouvance II, soixantedix mots pour le paysage, Paris, Edition de la Villette, $120 \mathrm{p}$.

Augoyard J.-F. (2011), Faire une ambiance, Paris, Éditions A la croisée, collection Ambiances, Ambiance, $527 \mathrm{p}$.

Bailly E. (2015), « L'appréhension du paysage urbain, une opportunité pour renouveler les conceptions urbaines environnementales et les démarches participatives ", in Luginbühl Yves (dir.), Biodiversité, paysage et cadre de vie, La démocratie en pratique, Paris, Editions Victoires, pp. 139-154.

Bailly E. Wakeman R., Duret H., Paquot T., Prié V. (2014), "L'enjeu du paysage commun ». Rapport de recherche du Programme de recherche Paysage et Développement Durable 2, Paris, MEDDE, $120 \mathrm{p}$.

Bailly E. (2013), " Des espaces publics aux espaces paysagers de la ville durable ", Articulo Journal of Urban Research, hors-série $n^{\circ} 4$, en ligne : http://articulo.revues.org/2233

Bailly E. (2009), Espaces imaginés, espaces habités : au-delà de la mondialisation - Téhéran, Rabat, Paris et New York, thèse de doctorat, sous la direction de Thierry Paquot, Institut d'Urbanisme de Paris, 450p.

Berleant A. (1990), The Aesthetics of Environment, Philadelphia, Temple University Press, $315 \mathrm{p}$.

Bernard J. (2015), "Les voies d'approches des émotions, enjeu de définition et catégorisations ", Terrains/Théories, n²/2015, en ligne : http://teth.revues.org/196

Berque A. (dir.) (1994). Cinq propositions pour une théorie du paysage, Seyssel, Champ Vallon, $122 \mathrm{p}$.

Berque A. (1996), Etre humain sur terre, Paris, Gallimard, 212 p.

Berthoz A. (1997), Le Sens du mouvement, Paris, Editions Odile Jacob, 336 p. 
Blanc N. (2012), Les nouvelles esthétiques urbaines, Paris, Armand Colin, 220 p.

Blanc N. et Lolive J. (2013), " Esthétique environnementale et projet paysager participatif », in Luginbühl Y. (coord.), Paysage et Développement Durable, Paris, Editions Quae, pp. 247265.

Boltanski L. (1993), La souffrance à distance. Morale humanitaire, médias et politique, Paris, Éditions Métailié, 288 p.

Bourdieu P. (1987), Choses dites, Paris, Editions de Minuit, 228 p.

Brinckerhoff J. J. (1984), A la découverte du paysage vernaculaire, New Haven et Londres, Yale University Press, réédition : Paris, Acte Sud, 277 p.

De Certeau M. (1990), L'Invention du quotidien, Paris, Folio, collection Essai, 416 p.

Dewey J. (1929), The Quest for Certainty, New-York, Minton, Balch \& Co, 335 p.

Durant G. (1996), Introduction à la mythologie, Paris, Albin Michel, 256 p.

Feildel B. (2010), Espaces et projets à l'épreuve des affects. Pour une reconnaissance du rapport affectif à l'espace dans les pratiques d'aménagement et d'urbanisme, thèse de doctorat, Tours, Université François Rabelais. https://tel.archives-ouvertes.fr/tel-00537920 Feildel B. (2013), « Vers un urbanisme affectif. Pour une prise en compte de la dimension sensible en aménagement et en urbanisme ", Norois, $n^{\circ} 227$, pp. 55-68, en ligne: https://norois.revues.org/4674

Florida R. (2005), Cities and the creative class, New York et Londres, Routledge, 198 p.

Foucault M. (1967), "Des espaces autres, Hétérotopies ", conférence au Cercle d'études architecturales, 14 mars 1967, in Architecture, Mouvement, Continuité, n5, octobre 1984, pp. 46-49.

Gibson J.J. (1979), The Ecological Approach to Visual Perception, Boston, Houghton Mifflin, $332 \mathrm{p}$.

Goffman E. (1963), Behaviour in public places. Notes on the social organization of gatherings. New York, Free Press, Reissue Edition, 248 p.

Jeudy H.P. (2003), Critique de l'esthétique urbaine, Paris, Sens et Tonka, 240 p.

Ledrut R. (1984), La forme et le sens dans la société, Paris, Librairie des méridiens, 192 p.

Duany A., Lyndon M., Speck J.(2010), The smart growth manual, United States, McGraw-Hill Education, $240 \mathrm{p}$.

Livet P. et Thévenot L. (1997), « Modes d'action collective et construction éthique; les émotions dans l'évaluation ", in Dupuy, J.-P. et Livet P., Les limites de la rationalité, tome 1 , Rationalité, éthique et cognition, Paris, La Découverte, pp. 412-439.

Luginbühl Y. (2012), La mise en scène du monde. Construction du paysage européen, Paris, CNRS Editions, $432 \mathrm{p}$.

Manola T. (2013), " L'implication habitante dans les quartiers dits durables: vers une participation paysagère ? ", Articulo - Journal of Urban Research, hors-série $n^{\circ} 4$, en ligne : https://articulo.revues.org/2251

Martouzet D. (2013), "Introduction. Une ville, cinq sens, trois traitements : sensoriel, cognitif et affectif ", Norois, $n^{\circ} 227$, pp. 7-10, en ligne : https://norois.revues.org/4635

Martouzet D. (2014), Ville aimable, Tours, Presses Universitaires François Rabelais, collection Villes et territoires, $384 \mathrm{p}$.

Mauss M. (1934), "Les techniques du corps ", in Sociologie et anthropologie (1960), Paris, Presses Universitaires de France, pp. 363-386.

Rancière J. (2000), Partage du sensible, Esthétique et politique, Paris, La fabrique éditions, $74 \mathrm{p}$.

Reddy W. M. (2001), The Navigation of Feeling. A Framework for the History of Emotions, 
Cambridge, Cambridge University Press, 380 p.

Sansot P. (2004), Poétique de la ville, Paris, Payot, collection Petite Bibliothèque Payot, $640 \mathrm{p}$.

Simmel G. (2013) Les grandes villes et la vie de l'esprit. Suivi de "Sociologie des sens ", Paris, Payot, collection Petite Bibliothèque Payot, $107 \mathrm{p}$.

Thibault J. P. (2015), "The backstage of urban ambiances », Emotion, Space et Society, vol. 15, pp.39-46, en ligne : https://www.researchgate.net/publication/264426664 The backstage of urban ambianc es When atmospheres pervade everyday experience Tuan Y.-F. (1977), Space and Place: The perspective of Experience, Minnesota, Edition University of Minnesota Press, 248 p.

Weber M. (1922), Economie et société, Paris, Poche, collection Evolution, 410 p.

Zirotti J.P. (2010), "Sociologie de l'action et émotions. Les émotions dans l'expérience du déni de citoyenneté chez les jeunes de banlieue ", Noesis, ${ }^{\circ} 16$, pp.47-62, en ligne: http://noesis.revues.org/1721 